\title{
Radio Supernovae in the Great Survey Era
}

\author{
Amy Lien, Nachiketa Chakraborty, Brian D. Fields ${ }^{1}$, Athol Kemball \\ Department of Astronomy, University of Illinois, Urbana, IL
}

\begin{abstract}
Radio properties of supernova outbursts remain poorly understood despite longstanding campaigns following events discovered at other wavelengths. After $\sim 30$ years of observations, only $\sim 50$ supernovae have been detected at radio wavelengths, none of which are Type Ia. Even the most radio-loud events are $\sim 10^{4}$ fainter in the radio than in the optical; to date, such intrinsically dim objects have only been visible in the very local universe. The detection and study of radio supernovae (RSNe) will be fundamentally altered and dramatically improved as the next generation of radio telescopes comes online, including EVLA, ASKAP, and MeerKAT, and culminating in the Square Kilometer Array (SKA); the latter should be $\gtrsim 50$ times more sensitive than present facilities. SKA can repeatedly scan large $\left(\gtrsim 1 \mathrm{deg}^{2}\right)$ areas of the sky, and thus will discover RSNe and other transient sources in a new, automatic, untargeted, and unbiased way. We estimate SKA will be able to detect core-collapse RSNe out to redshift $z \sim 5$, with an all-redshift rate $\sim 620$ events $\mathrm{yr}^{-1} \mathrm{deg}^{-2}$, assuming a survey sensitivity of $50 \mathrm{nJy}$ and radio lightcurves like those of SN 1993J. Hence SKA should provide a complete core-collapse RSN sample that is sufficient for statistical studies of radio properties of core-collapse supernovae. EVLA should find $\sim 160$ events $\mathrm{yr}^{-1} \mathrm{deg}^{-2}$ out to redshift $z \sim 3$, and other SKA precursors should have similar detection rates. We also provided recommendations of the survey strategy to maximize the RSN detections of SKA. This new radio core-collapse supernovae sample will complement the detections from the optical searches, such as the LSST, and together provide crucial information on massive star evolution, supernova physics, and the circumstellar medium, out to high redshift. Additionally, SKA may yield the first radio Type Ia detection via follow-up of nearby events discovered at other wavelengths.
\end{abstract}

\footnotetext{
${ }^{1}$ Department of Physics, University of Illinois, Urbana, IL
} 


\section{Introduction}

Supernovae are among the most energetic phenomena in the universe, and are central to cosmology and astrophysics. For example, core-collapse supernovae are explosions arise from the death of massive stars and hence are closely related to the cosmic star-formation rate and to massive-star evolution, and are responsible for the energy and baryonic feedback of the environment (Madau et al. 1998). Type Ia supernovae show a uniform properties in their lightcurves and play a crucial role as cosmic "standardizable candles" (Phillips 1993; Riess et al. 1996).

Our knowledge of the optical properties of supernovae, is increasing rapidly with the advent of prototype "synoptic"-i.e., repeated scan-sky surveys, such as SDSS-II (Frieman et al. 2008; Sako et al. 2008) and SNLS (Bazin et al. 2009; Palanque-Delabrouille et al. 2010). These campaigns are precursors to the coming "Great Survey" era in which synoptic surveys will be conducted routinely over very large regions of sky, e.g., LSST (The LSST Collaboration 2007; LSST Science Collaborations et al. 2009) and Pan-STARRS (Tonry 2003). The number of detected supernovae will increase by several orders of magnitude in this era (LSST Science Collaborat 2009; Lien \& Fields 2009).

In contrast to this wealth of optical information, properties of supernovae in the radio remain poorly understood, fundamentally due to observational limitations. Radio supernovae (RSNe) have primarily been discovered by follow-up observations of optical outbursts, and only very rarely by accident. To date, only $\sim 50$ core-collapse outbursts have radio detections, and no Type Ia explosion has ever been detected in the radio (Weiler et al. 2004; Panagia et al. 2006). The core-collapse subtype Ibc has been a focus of recent study in the radio, because some Type Ibc events are associated with long Gamma-Ray Bursts (GRBs) (Galama et al. 1998; Kulkarni et al. 1998; Soderberg 2007; Berger et al. 2003).

Current radio interferometers are scheduled primarily around targeted observations proposed by individual principal investigators. This stands in contrast to future radio interferometers planned for the coming "Great Survey" era. These include the Square Kilometer Array (SKA 1 ) and its precursor prototype arrays (for example, ASKAP2 and MeerKAT 3 ). These telescopes will operate primarily as wide-field survey instruments focusing on several key science projects (Carilli \& Rawlings 2004). As synoptic telescopes, they will be far better suited to study all classes of transient and time-variable radio sources, including RSNe.

\footnotetext{
${ }^{1}$ http://www.ska-telescope.org

${ }^{2}$ http://www.atnf.csiro.au/projects/askap

${ }^{3}$ http://www.ska.ac.za/meerkat
} 
Gal-Yam et al. (2006) already pointed out the power of synoptic radio surveys for detecting radio transients of various types, including supernovae and GRBs, in an unbiased way. Here we quantify the prospects for RSNe.

In this paper we explore this fundamentally new mode of untargeted RSN discovery and study. We adopt a forward-looking perspective, and consider the new science enabled by RSNe observations in an era in which the full SKA is operational. Our focus is mainly on core-collapse supernovae, the type for which some radio detections already exist. However, we will also discuss the possibility of Type Ia radio discovery based on current detection limits. We will first summarize current knowledge of radio core-collapse supernovae (\$2), and the expected sensitivity of SKA (\$3). Using this information, we forecast the radio core-collapse supernovae harvest of SKA (\$4), and consider optimal survey strategies (\$5). We conclude by anticipating the RSN science payoff in this new era (\$6]). We adopt a standard flat $\Lambda$ CDM model with $\Omega_{\mathrm{m}}=0.274, \Omega_{\Lambda}=0.726$, and $H_{0}=70.5 \mathrm{~km} \mathrm{~s}^{-1} \mathrm{Mpc}^{-1}$ (Komatsu et al. 2009) throughout.

\section{Radio Properties of Supernovae}

Several key properties of RSNe have been established, as a result of the longstanding leadership of the NRL-STScI group (recently reviewed in Weiler et al. 2009; Stockdale et al. 2007; Panagia et al. 2006) and of the CfA group and others (summarized in Soderberg 2007; Berger et al. 2003). We summarize these general RSN characteristics, which we will use to forecast the RSN discovery potential of synoptic radio surveys.

\subsection{Radio Core-Collapse Supernovae}

Observed core-collapse RSNe have luminosities spanning $\nu L_{\nu} \sim 10^{33}-10^{38} \mathrm{erg} \mathrm{s}^{-1}$ at 5 $\mathrm{GHz}$, and thus are $\gtrsim 10^{4}$ times less luminous in the radio than in the optical. Their intrinsic faintness has prevented RSN detection in all but the most local universe. Even within a particular core-collapse subtype, radio luminosities and lightcurves are highly diverse, e.g., two optically similar Type Ic events might be radio bright in one case and undetectable in the other (Munari et al. 1998; Nakano \& Aoki 1997; Stockdale et al. 2006) 4. Additionally, core-collapse RSNe spectral shapes strongly evolve with time; lightcurves peak over days to

\footnotetext{
${ }^{4}$ New Radio Supernova Results (Stockdale et al. 2006) are available online at: http://rsdwww.nrl.navy.mil/7213/weiler/sne-home.html
} 
months depending on the frequency. RSN emission can be understood in terms of interactions between the blast, ambient relativistic electrons, and the circumstellar medium (Chevalier 1982a, b, 1998).

To model RSN emission as a function of frequency and time, we adopt the semi-empirical form derived by Chevalier (1982a) and extended in Weiler et al. (2002),

$$
L(t, \nu)=L_{1}\left(\frac{\nu}{5 \mathrm{GHz}}\right)^{\alpha}\left(\frac{t}{1 \text { day }}\right)^{\beta} e^{-\tau_{\text {external }}}\left(\frac{1-e^{-\tau_{\mathrm{CSM}_{\mathrm{clumps}}}}}{\tau_{\mathrm{CSM}_{\mathrm{clumps}}}}\right)\left(\frac{1-e^{-\tau_{\text {internal }}}}{\tau_{\text {internal }}}\right)
$$

We follow the notation of Weiler et al. (2002). $L(t, \nu)$ is the supernova luminosity at frequency $\nu$ and time $t$ after the explosion. Optical depths from material both outside $\left(\tau_{\text {external, }}\right.$, $\left.\tau_{\mathrm{CSM}_{\text {clumps }}}\right)$ and inside $\left(\tau_{\text {internal }}\right)$ the blast-wave front are taken into account (see Weiler et al. 2002).

Parameters embedded in each optical depth term are those for SN 1993J, one of the best studied RSNe (Weiler et al. 2007). Radio emission from SN 1993J is dominated by the clumped-circumstellar-medium (clump-CSM) term, and hence

$$
L(t, \nu) \sim \frac{1-e^{-\tau_{\mathrm{CSM}_{\mathrm{clumps}}}}}{\tau_{\mathrm{CSM}_{\mathrm{clumps}}}}
$$

where $\tau_{\mathrm{CSM}_{\text {clumps }}}=4.6 \times 10^{5}\left(\frac{\nu}{5 \mathrm{GHz}}\right)^{-2.1}\left(\frac{t}{1 \text { day }}\right)^{-2.83}$, for SN 1993J. At small $t, \tau_{\mathrm{CSM}_{\text {clumps }}}$ is large and $L(t, \nu) \sim 1 / \tau_{\mathrm{CSM}_{\text {clumps }}} \propto \nu^{2.1} t^{2.83}$, so luminosity grows as a power law at early times. With all optical depth parameters fit to SN 1993J, the peak luminosity is controlled by the prefactor $L_{1}$.

Our main focus will be on RSN discovery, and thus it is most important to capture the wide variety of peak radio luminosities, which correspond in our model to a broad distribution for $L_{1}$. Figure 1 shows a crude luminosity function (not-normalized) based on the sample of 20 core-collapse supernovae (15 Type II and 5 Type Ibc) that have a published peak luminosity at $5 \mathrm{GHz}$ (Weiler et al. 2004; Stockdale et al. 2003, 2007; Papenkova et al. 2001; Stockdale et al. 2006; Baklanov et al. 2005; Pooley et al. 2002). We use $5 \mathrm{GHz}$ data to construct our luminosity function because the most RSNe have been observed at this frequency. However, our predictions will span a range of frequencies, based on this luminosity function and eq. (2). The data are divided into four luminosity bins of size $\Delta \log _{10}(L)=1$. The black curve in Fig. 1] is the best-fit Gaussian, with average luminosity $\log _{10}\left(L_{\mathrm{avg}} / \mathrm{erg} \mathrm{s}^{-1} \mathrm{~Hz}^{-1}\right)=27.3$, a standard deviation $\sigma=1.25$, and $\chi^{2}=0.18$. SN 1987A is marked in Fig. 1, but was not used in the fit to avoid possible bias due to its uncommonly low luminosity. The fitted luminosity function might be biased towards the brighter end, because of the current survey sensitivity and the small and incomplete nature of the sample. 


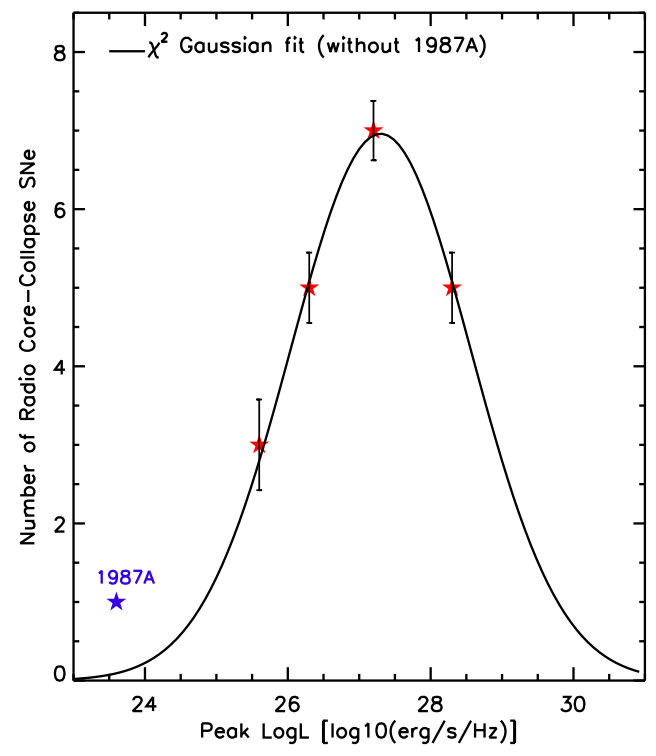

Fig. 1.- Radio luminosity function (not-normalized) at $5 \mathrm{GHz}$ of core-collapse supernovae showing core-collapse supernovae count as a function of $\log _{10}(L)$, where $\mathrm{L}$ is the peak radio luminosity. Data are binned to $\Delta \log _{10}(L)=1$. The black curve shows the $\chi^{2}$-fitted Gaussian to the underlying data (red stars).

\subsection{Radio Type Ia Supernovae}

All searches to date have failed to detect radio emission from Type Ia supernovae. Panagia et al. (2006) reported the radio upper limits of 27 Type Ia supernovae from more than two decades of observations by the Very Large Array (VLA). The weakest limit on a Type Ia event is $4.2 \times 10^{26} \mathrm{erg} \mathrm{s}^{-1} \mathrm{~Hz}^{-1}$ at $1.5 \mathrm{GHz}$ for $\mathrm{SN} 1987 \mathrm{~N}$, which is around one order of magnitude lower than the average luminosity of radio-detected core-collapse supernovae (see \$2.1). The strongest limit on Type Ia radio emission is even tighter, $8.1 \times 10^{24} \mathrm{erg} \mathrm{s}^{-1} \mathrm{~Hz}^{-1}$ at $8.3 \mathrm{GHz}$ for SN 1989B. Additionally, the $z \sim 0$ cosmic Type Ia supernova rate is around 1/4.5 of the core-collapse supernova rate (Bazin et al. 2009). The intrinsic faintness in radio and their smaller rate make detecting Type Ia in radio observations especially hard.

\section{Next-Generation Radio Telescopes: Expected Sensitivity}

Radio detections of supernovae to date have been restricted both by the limiting sensitivity of contemporary radio interferometers and the need for dedicated telescope time for 
transient followup. This situation will change drastically with SKA's unprecedented sensitivity and particularly by its ability to repeatedly scan large regions of the sky at this great depth.

Current SKA specifications adopt a target sensitivity parameter $A_{\text {eff }} / T_{\text {sys }}=10^{4} \mathrm{~m}^{2} \mathrm{~K}^{-1}$ at observing frequencies in the low several $\mathrm{GHz}$, including $z=0 \mathrm{HI}$ at $1.4 \mathrm{GHz}$. $A_{\mathrm{eff}}$ is the effective aperture, and $T_{\text {sys }}$ is the system temperature. We will adopt this value of $A_{\text {eff }} / T_{\text {sys }}$, which yields a 1- $\sigma$ rms thermal noise limit in total intensity of

$$
\sigma_{I}=0.15 \mu \mathrm{Jy}(\Delta \nu / \mathrm{GHz})^{-1 / 2}(\delta \mathrm{t} / \mathrm{hr})^{-1 / 2},
$$

for a bandwidth $\Delta \nu$ and observation duration $\delta t$. The SKA will therefore reach a thermal noise limit of several nJy in deep continuum integrations $(\delta t \sim 1000 \mathrm{hr}$ ) (SKA Design Reference Mission 2009) 5. We define the associated survey sensitivity $S_{\min }$ (the minimum flux density threshold) as $S_{\min }=3 \sigma_{I}$. In common with other radio interferometers, SKA will accumulate sensitivity in targeted deep fields, including transient-monitoring fields, by accumulating integration time over multiple individual observing tracks. We therefore will adopt a fiducial SKA supernova sensitivity of $S_{\min }=50 \mathrm{nJy}$ in 100 hours of observation, but we will show how our results are sensitive to other choices of $S_{\min }$.

It is anticipated that transient fields will be revisited with a cadence appropriate to the variability timescales under study and that interferometric inverse imaging methods will include source models with time variability. Survey optimization for interferometric transient detection is an active area of current SKA research. The technical details are beyond the intent and scope of this paper, but will be influenced by science goals for transient source study in general, including pulsars, GRBs, and supernovae (as considered in this paper), as well as the as-yet undiscovered transient population.

\section{Radio Supernovae for SKA}

With its unprecedented sensitivity, SKA will be capable of synoptic search for corecollapse RSNe and open new possibilities in radio astronomy. In this section, we predict the RSN detections of SKA based on current knowledge to demonstrate how the RSN survey can be done.

\footnotetext{
${ }^{5}$ http://www.skatelescope.org/PDF/DRM_v1.0.pdf
} 


\subsection{Core-Collapse Supernovae}

The detection rate $\Gamma_{\text {detect }}=d N_{\mathrm{SN}} /\left(d t_{\mathrm{obs}} d \Omega d z\right)$ for a given RSN survey is

$$
\Gamma_{\text {detect }}=f_{\text {survey }} f_{\text {radio }} f_{\mathrm{ISM}} \Gamma_{\mathrm{SN}}
$$

and is set by several observability factors $f$ that modulate the total rate of all supernovae

$$
\Gamma_{\mathrm{SN}}(z)=\frac{d N_{\mathrm{SN}}}{d V_{\text {comov }} d t_{\mathrm{em}}} \frac{d t_{\mathrm{em}}}{d t_{\mathrm{obs}}} \frac{d V_{\text {comov }}}{d \Omega d z}=\mathcal{R}_{\mathrm{SN}}(z) r_{\mathrm{comov}}^{2}(z) c\left|\frac{d t}{d z}\right|
$$

within the cosmic volume out to redshift $z$ (Madau et al. 1998; Lien \& Fields 2009).

We see that the total cosmic supernova rate $\Gamma_{\mathrm{SN}}$ depends on cosmology via the volume element and the time dilation terms. Because $\Lambda \mathrm{CDM}$ cosmological parameters are now known to high precision, these factors have a negligible error compared to the other ingredients in the calculation. The other factor in $\Gamma_{\mathrm{SN}}$ is the cosmic core-collapse supernova rate density $\mathcal{R}_{\mathrm{SN}}(z)=d N_{\mathrm{SN}} /\left(d V_{\text {comov }} d t_{\text {emit }}\right)$. Some direct measurements of this rate now exist out to $z \sim 1$, but the uncertainties remain large (Cappellaro et al. 1999; Dahlen et al. 2004; Cappellaro et al. 2005; Hopkins \& Beacom 2006; Botticella et al. 2008; Dahlen et al. 2008; Kistler et al. 2008; Bazin et al. 2009; Smartt et al. 2009; Dahlen et al. 2010; Li et al. 2011b; Horiuchi et al. 2011). However, core-collapse events are short-lived, and so the cosmic core-collapse rate is proportional to the cosmic star-formation rate $\dot{\rho}_{\star}$, which is much better-determined and extends to much higher redshifts. We thus derive $\mathcal{R}_{\mathrm{SN}}$ from the recent Horiuchi et al. (2009) fit to the cosmic star-formation rate. The proportionality follows from the choice of initial mass function; we apply the Salpeter initial mass function (Salpeter 1955) and assume the mass range of core-collapse SNe progenitors to be $8 M_{\odot}-50 M_{\odot}$; this gives $\mathcal{R}_{\mathrm{SN}}=\left(0.007 M_{\odot}^{-1}\right) \dot{\rho}_{\star}$

Several effects reduce the total rate $\Gamma_{\mathrm{SN}}$ to the observed rate $\Gamma_{\text {detect }}$ in eq. (44). Due to finite survey sensitivity, only a fraction $f_{\text {survey }}$ of events are bright enough to detect, and only some fraction $f_{\text {radio }}$ of supernovae will emit in the radio. We neglect interstellar extinction and assume $f_{\text {ISM }} \sim 1$ at the radio wavelengths considered.

The term $f_{\text {radio }}$ in eq. (41) contains the greatest uncertainty due to the relatively small sample of RSNe observed to date, and the unavoidable incompleteness of the sample (K. Weiler, private communication 2010). The only published fraction available is for Type Ibc supernovae. Using VLA for radio follow-up, Berger et al. (2003) suggests that $f_{\text {radio,Ibc }} \sim 12 \%$ after surveying 33 optically-detected Type Ibc supernovae. For the purpose of demonstration, we will adopt $f_{\text {radio }}=10 \%$ for the calculations presented in this paper, which we believe is rather conservative. 
An order-of-magnitude calculation provides a useful estimate of the expected corecollapse RSN rate. As discussed in $\$ 3$, we adopt a fiducial SKA sensitivity of $S_{\min }=$ $50 \mathrm{nJy}$. Hence SKA will be able to detect supernovae with average radio luminosity $(L \sim$ $10^{27} \mathrm{erg} \mathrm{s}^{-1} \mathrm{~Hz}^{-1}$ ) to a distance $D_{L}=\sqrt{L / 4 \pi S_{\min }} \sim 4 \mathrm{Gpc}$, which for a $\Lambda$ CDM cosmology corresponds to $z \sim 1$. This will give a detectable volume of $V_{\text {detect }} \sim(4 / 3) \pi D_{L}^{3} \sim 2.85 \times$ $10^{11} \mathrm{Mpc}^{3}$. Observations show that the core-collapse supernova rate $R_{\mathrm{SN}} \sim 10^{-3} \mathrm{yr}^{-1} \mathrm{Mpc}^{-3}$ at $z \sim 1$ (Dahlen et al. 2008, 2010). Assuming the fraction of the total core-collapse supernovae that display the adopted average radio luminosity to be $f_{\text {radio }} \sim 10 \%$ (Berger et al. $2003)$, the all-sky detection rate $d N_{\mathrm{SN}} / d t \sim R_{\mathrm{SN}} \times f_{\text {radio }} \times V_{\text {detect }} \sim 2.85 \times 10^{7} \mathrm{yr}^{-1}$. This corresponds to a areal detection rate $d N_{\mathrm{SN}} /(d t d \Omega) \sim 700 \mathrm{yr}^{-1} \mathrm{deg}^{-2}$. As we now see, a more careful calculation confirms this estimate.

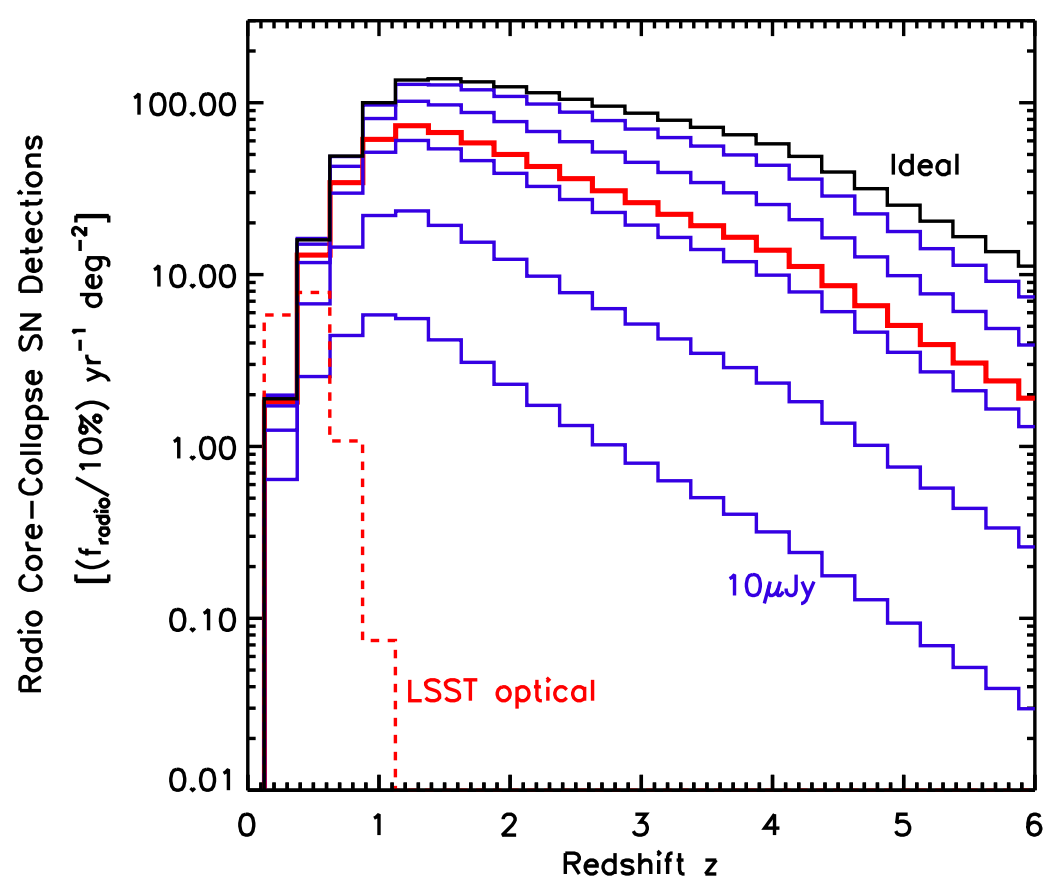

Fig. 2.- Estimated radio core-collapse supernova detection rate as a function of redshift at $1.4 \mathrm{GHz}$, assuming $f_{\text {radio }}=10 \%$. Predictions are shown for different survey sensitivities: $S_{\min }=\{10 \mu \mathrm{Jy}$ (blue), $1 \mu \mathrm{Jy}$ (blue), $100 \mathrm{nJy}$ (blue), $50 \mathrm{nJy}$ (thick red), $10 \mathrm{nJy}$ (blue), 1 nJy (blue) $\}$ from bottom to top solid curves, respectively. We adopt $50 \mathrm{nJy}$ as our benchmark sensitivity hereafter. For comparison, the red-dashed curve shows the LSST optical supernova detection rate per year per $\operatorname{deg}^{2}$ (Lien \& Fields 2009). Also, the top solid curve (black) plots the ideal core-collapse RSN rate for comparison. 


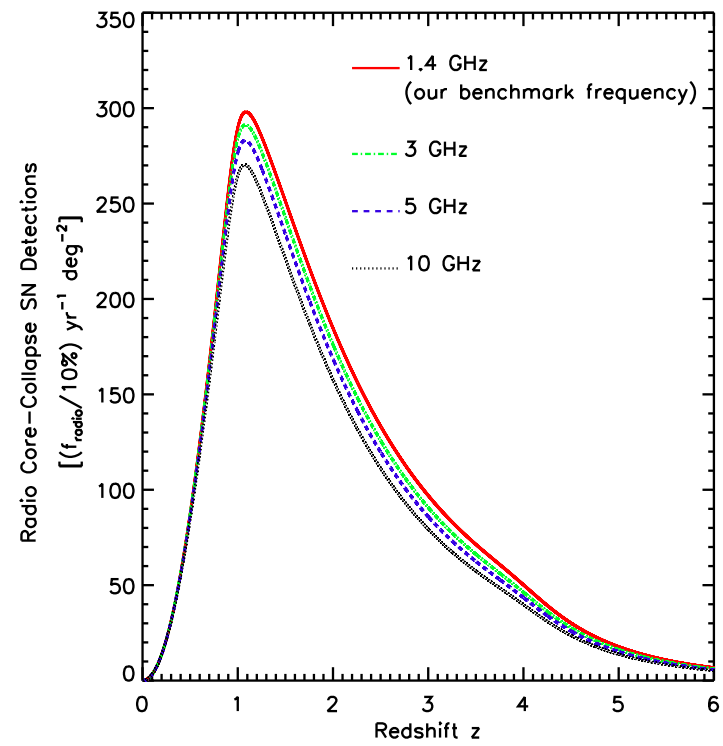

Fig. 3.- Estimated radio core-collapse supernova detection rate as a function of redshift for different frequency bands, for $f_{\text {radio }}=10 \%$, and an adopted survey sensitivity $S_{\min }=50$ nJy.
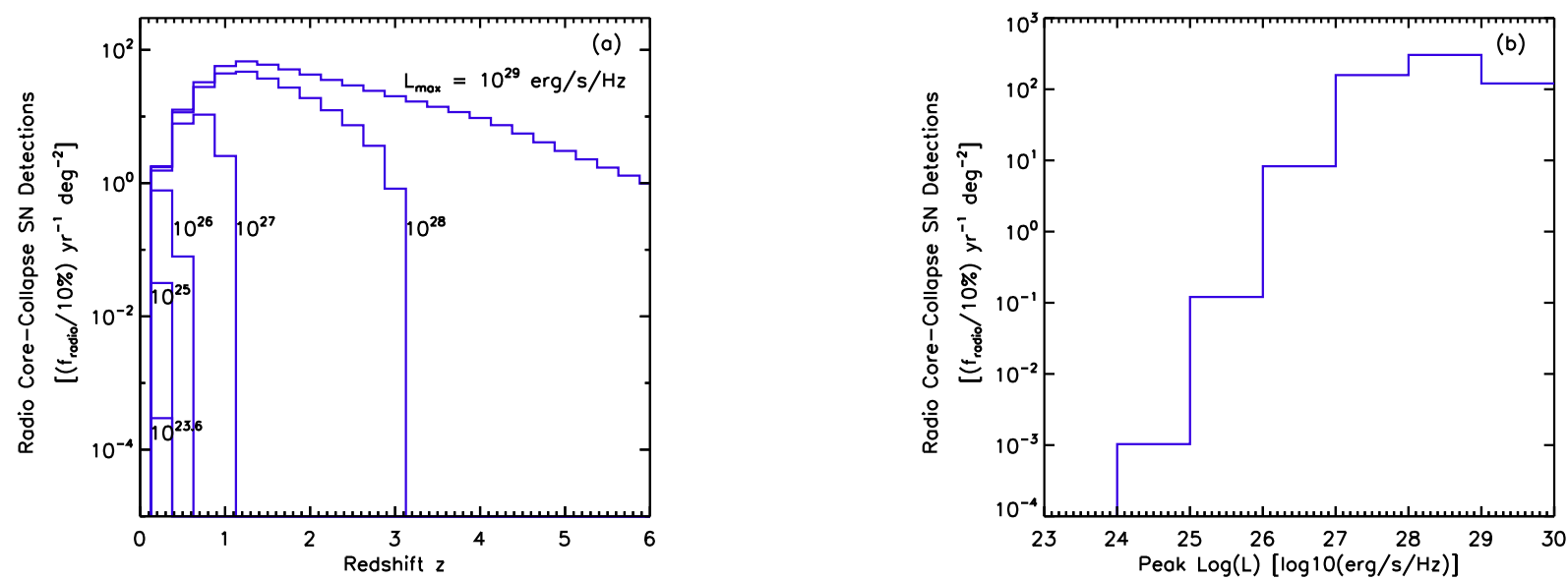

Fig. 4.- Core-collapse detection sensitivity to supernova radio luminosity, at $1.4 \mathrm{GHz}$, and for survey sensitivity $S_{\min }=50 \mathrm{nJy}$. (a)Left Panel: Supernova distribution over redshift, for different cutoffs for the luminosity function. (b)Right Panel: Supernova distribution in luminosity bins, integrated over all redshifts. 
A careful prediction involves detailed calculation of $f_{\text {survey }}(z)$. The fraction $f_{\text {survey }}(z)$ of observable radio-emitting events depends on adopted survey sensitivity, and on the normalized supernova luminosity function $\Phi_{5 \mathrm{GHz}}(\log L)$, which is measured at a peak luminosity at $5 \mathrm{GHz}$ (derived in §2). In this paper we will only consider whether a supernova is detectable at its peak luminosity at each corresponding frequency. The peak radio luminosity should be reached earlier at higher frequencies because of preferential absorption at lower frequencies (Weiler et al. 2002). At different redshift, the peak flux density $S_{\text {min }}^{\text {peak }}$ in the observed frequency $\nu$ can be tied to the corresponding luminosity threshold $L_{\min }^{\text {peak }}$ by

$$
L_{\mathrm{min}}^{\mathrm{peak}}\left(z ; \nu_{\mathrm{emit}}\right)=\frac{4 \pi D_{L}^{2}(z)}{(1+z)} S_{\mathrm{min}}^{\mathrm{peak}}\left(\nu_{\mathrm{obs}}\right),
$$

where the luminosity distance is $D_{L}(z)=(1+z) c / H_{0} \int_{0}^{z} d z^{\prime}\left[\Omega_{\mathrm{m}}\left(1+z^{\prime}\right)^{3}+\Omega_{\Lambda}\right]^{-1 / 2}$. However, because the luminosity function we used is based on the peak luminosity at $5 \mathrm{GHz}$, we must find the corresponding luminosity threshold at this frequency by applying corrections based on the radio spectrum,

$$
L_{\mathrm{min}, 5 \mathrm{GHz}}^{\text {peak }}=L_{\mathrm{min}}^{\text {peak }} \frac{\int_{5 \mathrm{GHz} \text { band }} S^{\text {peak }}\left(\nu_{\mathrm{em}}\right) d \nu_{\mathrm{em}}}{\int_{\text {obs band }} S^{\text {peak }}\left[(1+z) \nu_{\mathrm{obs}}\right] d \nu_{\mathrm{obs}}} .
$$

The detectable fraction resulting from survey sensitivity can therefore be estimated as

$$
f_{\text {survey }}=\int_{\log L_{\min , 5 \mathrm{GHz}}^{\text {peak }}} \Phi_{5 \mathrm{GHz}}(\log L) d \log L .
$$

Figure. 2 plots the results of our predicted core-collapse RSN detection rate for different target survey sensitivities, $S_{\min }$. We adopt a benchmark frequency of $1.4 \mathrm{GHz}$ because this will be one of the first major bands SKA deploys to observe neutral hydrogen. The related instantaneous field-of-view at $1.4 \mathrm{GHz}$ of current SKA designs based on dish reflectors is approximately $1 \mathrm{deg}^{2}$, which we adopt. Fig. 2 plots the ideal core-collapse supernova rate for comparison (assuming infinite sensitivity but $f_{\text {radio }}=10 \%$ ). One can see that the detection rate at $1 \mathrm{nJy}$ is very close to the ideal rate in the universe.

Results for our fiducial SKA flux limit $S_{\min }=50 \mathrm{nJy}$ are highlighted in Fig. 2. At this sensitivity, we see that we can expect that radio supernovae will be discovered (event rates $>5 \mathrm{RSN} \mathrm{yr}^{-1} \mathrm{deg}^{-2}$ ) over the enormous redshift range $z \simeq 0.5$ to 5 . The total rate of RSNe expected in this entire redshift range is

$$
\frac{d N_{\mathrm{SN}}}{d t d \Omega}(>50 \mathrm{nJy}) \approx 620 \mathrm{RSNeyr}^{-1} \mathrm{deg}^{-2},
$$

in good agreement with our above order-of-magnitude estimate. This sample size is large enough to be statistically useful and to allow for examination of the redshift history of RSNe. 
Moreover, out to $z \sim 1$, SKA will detect almost all cosmic RSNe in the field of view, while at higher redshift the detections still comprise $>10 \%$ of the underlying ideal cosmic rate. For comparison, we also see that LSST will detect optical supernovae out to $z \lesssim 1$. Thus SKA will be complementary to LSST as a unique tool for cosmic supernova discovery.

Figure. 3 shows how core-collapse RSN detections vary for different observing frequencies, fixing a common survey sensitivity $S_{\min }=50 \mathrm{nJy}$ and bandwidth $\Delta \nu=1 \mathrm{GHz}$. Results show similar numbers of detections at different bands, which is caused by a relatively flat spectrum shape at peak luminosities. Because SKA will be able to detect core-collapse RSNe out to high redshift $z \sim 5$, the frequency-redshift and time-dilation effects are significant. Weiler et al. (2002) noted that RSNe peak when the optical depth $\tau \sim 1$. Since the optical depth depends both on frequency and time with similar power index (Weiler et al. 2002), the frequency-redshift and time-dilation effects approximately cancel, so that a fixed observed frequency, the peak time is nearly redshift-independent.

As mentioned above, our luminosity function is likely biased toward the available bright events in a small and incomplete sample. To explore how this bias could affect our results, Fig. 4 shows how the detection rate with $S_{\min }=50 \mathrm{nJy}$ at $1.4 \mathrm{GHz}$ depends on corecollapse RSN luminosity. Fig. 4(a) shows that RSN with peak luminosities greater than $10^{27} \mathrm{erg} \mathrm{s}^{-1} \mathrm{~Hz}^{-1}$ contribute all of the detections beyond redshift $z \sim 1$, and RSN need to peak brighter than $10^{28} \mathrm{erg} \mathrm{s}^{-1} \mathrm{~Hz}^{-1}$ to be seen beyond $z \sim 3$. Fig. 4(b) similarly shows that the all-redshift detection rate becomes substantial for explosions peaking $>10^{26} \mathrm{erg} \mathrm{s}^{-1} \mathrm{~Hz}^{-1}$.

Type Ibc supernovae are of particular interest given their association with long gammaray bursts (GRBs; Galama et al. 1998; Woosley 1993; Heger et al. 2003). Fig. 5 shows our predictions for Type Ibc detections of SKA per year per $\operatorname{deg}^{2}$ at $1.4 \mathrm{GHz}$ with a survey sensitivity of $50 \mathrm{nJy}$. The red curve shows the radio Type Ibc detections, assuming that Type Ibc represents $25 \%$ of core-collapse events ( $\mathrm{Li}$ et al. 2011a), and $f_{\text {radio,Ibc }}=12 \%$ with luminosity $\sim 10^{27} \mathrm{erg} \mathrm{s}^{-1} \mathrm{~Hz}^{-1} 6$ (Berger et al. 2003). The blue curve shows the possible detections of the sub-class of Type Ibc supernovae that display extreme radio emission and hence might be powered by central engines and related to GRBs. We assume that $0.5 \%$ of all Type Ibc supernovae are powered by central engines and have luminosities of $\sim 10^{29} \mathrm{erg} \mathrm{s}^{-1} \mathrm{~Hz}^{-1}$ (Berger et al. 2003). We adopted the spectrum of SN 1998bw, which is a Type Ic supernova (Weiler et al. 2002). Under these assumptions the SKA will be able to make unbiased, untargeted detections of $\sim 130$ radio Type Ibc supernovae per year per $\mathrm{deg}^{2}$, and $\sim 20$ Type Ibc supernovae that might be connected to GRBs.

\footnotetext{
${ }^{6}$ Here we simply assume a Gaussian distribution for the luminosity function centered at the specified luminosity with $\sigma=1$.
} 


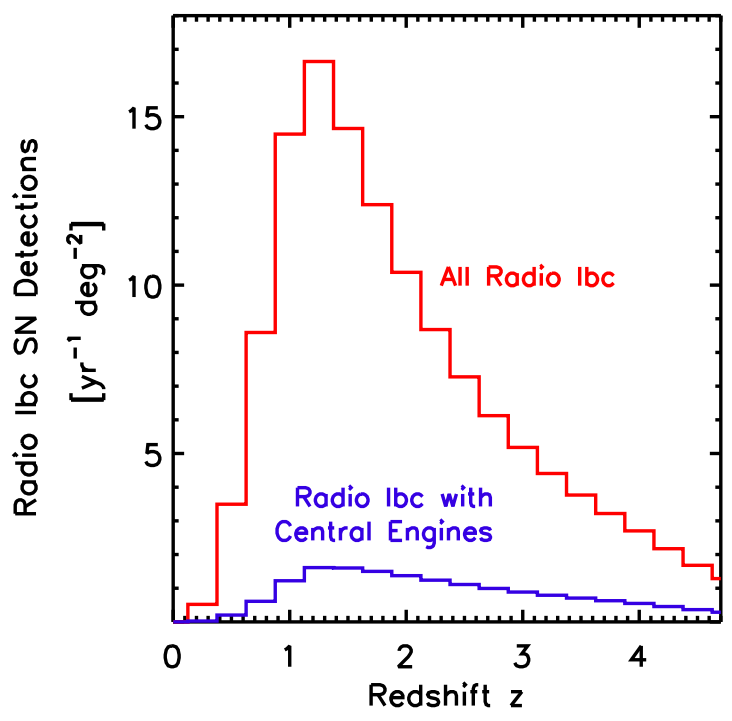

Fig. 5.- Predicted detection rate of Type Ibc supernovae as a function of redshift. In this plot we assume the sensitivity for SKA is $S_{\min }=50 \mathrm{nJy}$. The red curve shows all of the radio Ibc detections, assuming $f_{\text {radio,Ibc }}=12 \%$ (Berger et al. 2003). The blue curve shows only the detection rate for Radio Ibc with central engines, assuming $0.5 \%$ of all of the Type Ibc RSNe are powered by central engines.

Finally, we turn to SKA precursors. The EVLA7, a current leading-edge radio interferometer operating at centimeter wavelengths, is anticipated to reach a $1-\sigma$ rms noise of $\sigma_{I} \sim 1 \mu \mathrm{Jy}$ or less in 10 hours, while SKA is expected to reach $\sigma_{I} \sim 50 \mathrm{nJy}$ in 10 hours. With data accumulated over repeated scans spanning over 1000 hours, an rms $\sigma_{I} \sim 5$ nJy may be reached. In synoptic surveys, we would expect EVLA to detect core-collapse events

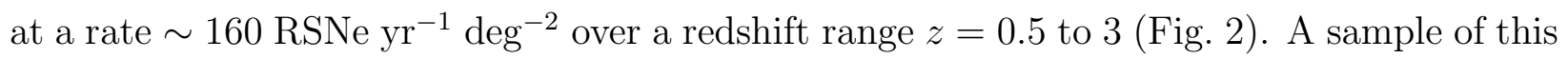
size over this redshift range will already mark a major advance in the study of cosmic RSNe, and further motivate the full SKA. ASKAP and MeerKAT are expected to have sensitivities comparable to that of EVLA (Johnston et al. 2009; de Blok et al. 2010), hence we would expect these to detect $\mathrm{RSNe}$ with similar rates and redshift reach.

\footnotetext{
${ }^{7}$ http://www.aoc.nrao.edu/evla
} 


\subsection{Type Ia Supernovae}

If all Type Ia RSNe are dimmer than the weakest limit presented in 92.2 , the expected SKA detection rate is essentially zero. For example, if a typical Type Ia has a radio luminosity equal to the lowest published limit, $L=8.1 \times 10^{24} \mathrm{erg} \mathrm{s}^{-1} \mathrm{~Hz}^{-1}$, this can be seen with a sensitivity $S_{\min }=50$ nJy out to a luminosity distance $\sim 300 \mathrm{Mpc}(z \sim 0.08)$. While $\sim 3900$ cosmic Ia events should occur per year out to this distance over the entire sky, $\ll 1$ events are expected in the SKA field of view. More optimistically, imagine a typical Type Ia radio luminosity is $L=10^{26} \mathrm{erg} \mathrm{s}^{-1} \mathrm{~Hz}^{-1}$, which is below $L=4.2 \times 10^{26} \mathrm{erg} \mathrm{s}^{-1} \mathrm{~Hz}^{-1}$, the highest published limit (Panagia et al. 2006); here the luminosity distance increases to $\sim 1400 \mathrm{Mpc}$ $(z \sim 0.28)$. In this case, we find an SKA Type Ia detection rate $\sim 0.5 \mathrm{yr}^{-1} \mathrm{deg}^{-2}$, based on the local cosmic Type Ia rate derived from SDSS-II optical data (Dilday et al. 2010), $S_{\min }=50 \mathrm{nJy}$, and $f_{\text {radio }}=10 \% 8$ We see that even optimistically, we expect fewer than one event per SKA field-of-view per year. Even with $f_{\text {radio }}=100 \%$, the detection rate is still only $\sim 5 \mathrm{yr}^{-1} \mathrm{deg}^{-2}$. Therefore we conclude that SKA will make few, if any, blind detections of Type Ia supernovae.

Targeted radio observations to follow up from nearby optical detections will probably be the best way to search for such events. For example, we expect 10 Type Ia events/year in the LSST sky within $\sim 60 \mathrm{Mpc}(z \sim 0.015)$. Type Ia (or core collapse!) events within this distance observed with $S_{\min }=50 \mathrm{nJy}$, would be detectable at luminosities $L \gtrsim 3.0 \times$ $10^{23} \mathrm{erg} \mathrm{s}^{-1} \mathrm{~Hz}^{-1}$. Amusingly, this is close to the radio luminosity of SN 1987A.

\section{Radio Survey Recommendations}

A key requirement for detecting weak radio emission from CSM-supernovae interactions is improved radio interferometer sensitivity. High angular resolution - below an arcsecond at $1.4 \mathrm{GHz}$, (Weiler et al. 2004) - is also required to avoid natural confusion and to help identify supernovae against background galaxies. This is similar to the maximum EVLA angular resolution at $1.4 \mathrm{GHz}$. For comparison, the maximum anticipated SKA baseline length of $3000 \mathrm{~km}$, producing angular resolution of 0.014 arcsecond at $1.4 \mathrm{GHz}$, is sufficient to distinguish different galaxies and also to resolve galaxies as extended sources within the observable universe with rms confusion limit of $<3$ nJy at $1.4 \mathrm{GHz}$ (Carilli \& Rawlings 2004).

A key science goal of the SKA is to detect transient radio sources, both known (e.g.

\footnotetext{
8 This also is implied by Fig. 4, which is for core-collapse events that have a higher cosmic rate.
} 
pulsars, GRBs), and as-yet unknown. This requires sophisticated transient detection and classification algorithms very likely running commensally with other large surveys planned by the SKA, such as the HI spectroscopic survey and deep continuum fields. We assume here that SKA transient detection algorithms will encompass automated detection of RSNe. For example, current parameterized models (Weiler et al. 1986, 1990; Montes et al. 1997; Chevalier 1982a, b) based on available data predict patterns of spectral index evolution characteristic of supernovae in general, and supernova sub-types in particular. This information could be exploited for RSN detection, even potentially against a background of unrelated source variability. Broad frequency coverage is important in this regard (Weiler et al. 2004).

The SKA intrinsically is a high dynamic-range instrument, given the sensitivity implied by the large collecting area. The most demanding SKA science applications will require a dynamic range of $10^{7}: 1$. The detection of faint RSNe will require a dynamic range that falls within that envelope.

Although the lightcurves of RSNe show great diversity, the luminosities of core-collapse supernovae usually change much slower in radio than in optical. RSN lightcurves typically evolve on timescales of weeks to years; a useful lightcurve compilation appears in Stockdale et al. (2007). Thus the minimum survey cadence (revisit periodicity) need not be any more frequent than this. Also, we have shown that core-collapse RSNe can be found out to high redshift with surveys pushing down to $S_{\min }=50 \mathrm{nJy}$. For SKA this corresponds to about 100 hours of exposure, in line with planned deep field exposures which are part of the key science. Thus, SKA as currently envisioned is well-suited to core-collapse discovery.

On the other hand, SKA probably will not have sufficient survey sensitivity for a volumetric search for Type Ia events, based on our current knowledge of the cosmic Type Ia rate and the upper limits in their luminosities set by the non-detection of these events. Follow-up observations from other wavelengths will likely be the best way to search for Type Ia RSNe.

The small volume of the local universe will limit nearby untargeted SKA detections of low-redshift core-collapse RSNe. We estimate only $\sim 2$ core-collapse RSN detections per year per square degree within redshift $z \sim 0.5$ (assuming a 50 nJy sensitivity at 1.4 $\mathrm{GHz}$ and $f_{\text {radio }}=10 \%$ ). Unless SKA has large sky coverage comparable to those of optical surveys, it will be hard to get statistical information from such a small sample. Therefore, targeted radio followup of optically-confirmed nearby supernovae will be crucial to build a core-collapse RSN "training set" database needed for refining automatic identification and classification techniques.

With detection methods optimized based on low- $z$ radio data for optically-identified events, radio surveys can then be used to independently detect core-collapse RSNe at high 
redshift based only on their radio emission. As shown in Fig. 2, supernova searches at high redshift $(z \gtrsim 1)$ will largely rely on radio synoptic surveys, the inverse of the strategy proposed above for low-redshift domain. Surveys for core-collapse RSNe will likely not be synoptic all-sky surveys due to operational limitations, but will likely proceed in a limited set of sub-fields, visited over an hierarchical set of cadences to cover a range of time-scales for general transient phenomena and multiple commensal science objectives. It is also important to match core-collapse RSNe survey sky coverage and cadence to that used in complementary optical surveys. LSST will repeatedly scan the whole sky every $\sim 3$ days. Thus a cadence $\sim 1$ week for RSNe sub-fields will be preferred for an SKA core-collapse supernova survey.

\section{Discussion and Conclusions}

SKA's capability for unbiased synoptic searches over large fields of view will revolutionize the discovery of radio transients in general and core-collapse RSNe in particular (Gal-Yam et al. 2006). The unprecedented sensitivity of SKA could allow detection of corecollapse RSNe out to a redshift $z \sim 5$. These detections will be unbiased and automatic in that they can occur anywhere in the large SKA field of view without need for targeting based on prior detection at other wavelengths. With SKA, the core-collapse RSN inventory should increase from the current number of several dozen to $\sim 620 \mathrm{yr}^{-1} \mathrm{deg}^{-2}$. EVLA should detect $\sim 160 \mathrm{yr}^{-1} \mathrm{deg}^{-2}$, and other SKA precursors should reap similarly large RSN harvests. In contrast, intrinsically dim RSNe such as Type Ia events and 1987A-like core-collapse explosions are unlikely to be found blindly. However, the SKA (and precursor) sensitivities will offer the possibility of detecting these events via targeted followup of discoveries by optical synoptic surveys such as LSST.

The science payoff of large-scale RSNe searches touches many areas of astrophysics and cosmology. We conclude with examples of possible science applications with the new era of RSN survey. However, the true potential of untargeted radio search is very likely beyond what we mention.

Non-prompt RSN emission requires the presence of circumstellar matter, so such surveys will probe this material and the pre-supernova winds producing it. For core-collapse supernovae, pre-supernova winds should depend on the metallicities of the progenitor stars (Leitherer et al. 1992; Kudritzki \& Puls 2000; Vink et al. 2001; Mokiem et al. 2007), and should be weaker in metal-poor environments with lower opacities in progenitor atmospheres. This effect should lead to correlations between RSN luminosity and host metallicity, as well as an evolution of the RSN luminosity function towards lower values at higher redshifts. For Type Ia supernovae, the mass-loss rate from the progenitors depends on the nature of the 
binary system, i.e., single or double degenerate (Nomoto et al. 1984; Iben \& Tutukov 1984; Webbink 1984). Radio detection of Type Ia supernovae will probe the mass and density profile of the surrounding environment and hence be valuable for studying Type Ia physics (Eck et al. 1995; Panagia et al. 2006; Chomiuk et al. 2011).

Large-scale synoptic RSNe surveys will complement their optical counterparts. While optical surveys such as LSST will provide very large supernova statistics at $z \lesssim 1$, radio surveys will be crucial for detections at higher redshifts. The nature and evolution of dust obscuration presents a major challenge for optical supernova surveys and supernova cosmology. Current studies suggest dust obscuration increases rapidly with redshift, but uncertainties are large. Mannucci et al. (2007) estimate that optical surveys may miss $\sim 60 \%$ of core-collapse supernovae and $\sim 35 \%$ of Type Ia supernovae at redshift $z \sim 2$. RSN observations, in contrast, are essentially unaffected by dust. Thus, high-redshift supernovae could be detected at radio wavelengths but largely missed in counterpart optical searches. Comparing supernova detections in both optical and radio will provide a new and independent way to measure dust dependence on redshift. In particular, SKA will be a powerful tool to directly detect supernovae in dust-obscured regions at large redshift, and therefore offer what may be the only means to study the total supernovae rate, star-formation, and dust behavior in these areas.

Additionally, radio surveys will reveal rare and exotic events. For example, some Type Ibc supernovae are linked to long GRBs (Galama et al. 1998; and see reviews in Woosley \& Bloom 2006; Gehrels et al. 2009), probably via highly relativistic jets powered by central engines and will manifest themselves in extremely luminous radio emission (Woosley 1993; Iwamoto et al. 1998; Li \& Chevalier 1999; Woosley et al. 1999; Heger et al. 2003). Thus one might expect radio surveys to preferentially detect more Type Ibc supernovae than other supernova types. An unbiased sample of Type Ibc RSNe will provide new information about the circumstellar environments of these explosions and thus probe the mass-loss effects believed to be crucial to the Ibc pre-explosion evolutionary path (Price et al. 2002; Soderberg et al. 2004, 2006; Crockett et al. 2007; Wellons \& Soderberg 2011); in addition, a large sample of Ibc RSNe will allow systematic study of the differences, if any, between those which do an do not host GRBs (Berger et al. 2003; Soderberg et al. 2006; Soderberg 2007).

Furthermore, radio surveys give unique new insight into a possible class of massive star deaths via direct collapse into black holes, with powerful neutrino bursts but no electromagnetic emission (MacFadyen \& Woosley 1999; Fryer 1999; MacFadyen et al. 2001; Heger et al. 2003). These "invisible collapses" can be probed by comparing supernovae detected electromagnetically and the diffuse background of cosmic supernova neutrinos (Lien et al. 2010, and references therein). By revealing dust-enshrouded SNe, radio surveys will make this com- 
parison robust by removing the degeneracy between truly invisible events and those which are simply optically obscured. Indeed, direct collapse events without explosions but with relativistic jets are candidates for GRB progenitors. A comparison among RSNe, optical supernovae, GRBs, and neutrino observations will provide important clues to the physics of visible and invisible collapses, and their relation with GRBs.

We thus believe that a synoptic survey in radio wavelengths will be crucial in many fields of astrophysics, for it will bring the first complete and unbiased RSN sample and systematically explore exotic radio transients. SKA will be capable of performing such an untargeted survey with its unprecedented sensitivity. Our knowledge of supernovae will thus be firmly extended into the radio and to high redshifts.

We thank Kurt Weiler, Christopher Stockdale, and Shunsaku Horiuchi for encouragement and illuminating conversations. We are also grateful to Joseph Lazio for helpful comments that have improved this paper.

\section{REFERENCES}

Baklanov, P. V., Blinnikov, S. I., \& Pavlyuk, N. N. 2005, Astronomy Letters, 31, 429

Bazin, G., et al. 2009, A\&A, 499, 653

Berger, E., Kulkarni, S. R., Frail, D. A., \& Soderberg, A. M. 2003, ApJ, 599, 408

Botticella, M. T., et al. 2008, A\&A, 479, 49

Cappellaro, E., Evans, R., \& Turatto, M. 1999, A\&A, 351, 459

Cappellaro, E., et al. 2005, A\&A, 430, 83

Carilli, C. L., \& Rawlings, S. 2004, New Astronomy Review, 48, 979

Chevalier, R. A. 1982, ApJ, 259, 302

Chevalier, R. A. 1982, ApJ, 259, L85

Chevalier, R. A. 1998, ApJ, 499, 810

Chomiuk, L., Soderberg, A. M., Chevalier, R., Badenes, C., \& Fransson, C. 2011, Bulletin of the American Astronomical Society, 43, \#304.05

Crockett, R. M., et al. 2007, MNRAS, 381, 835 
de Blok, E. W. J. G., Booth, R., Jonas, J., \& Fanaroff, B. 2010, ISKAF2010 Science Meeting

Dahlen, T., et al. 2004, ApJ, 613, 189

T. Dahlen et al., The Extended HST Supernova Survey: The Rates of Type Ia and CC SNe at high-z, in Arcetri Supernova Rates Workshop, 2008, http://www.arcetri.astro.it/ filippo/snrate08/Home.html

Dahlen, T., Strolger, L., \& Riess, A. G. 2010, Bulletin of the American Astronomical Society, 42, \#430.23

Dilday, B., et al. 2010, ApJ, 713, 1026

Eck, C. R., Cowan, J. J., Roberts, D. A., Boffi, F. R., \& Branch, D. 1995, ApJ, 451, L53

Frieman, J. A., et al. 2008, AJ, 135, 338

Fryer, C. L. 1999, ApJ, 522, 413

Galama, T. J., et al. 1998, Nature, 395, 670

Gal-Yam, A., et al. 2006, ApJ, 639, 331

Gehrels, N., Ramirez-Ruiz, E., \& Fox, D. B. 2009, ARA\&A, 47, 567

Heger, A., Fryer, C. L., Woosley, S. E., Langer, N., \& Hartmann, D. H. 2003, ApJ, 591, 288

Hopkins, A. M., \& Beacom, J. F. 2006, ApJ, 651, 142

Horiuchi, S., Beacom, J. F., \& Dwek, E. 2009, Phys. Rev. D, 79, 083013

Horiuchi, S., Beacom, J. F., Kochanek, C. S., Prieto, J. L., Stanek, K. Z., \& Thompson, T. A. 2011, arXiv:1102.1977

Iben, I., Jr., \& Tutukov, A. V. 1984, ApJS, 54, 335

Iwamoto, K., et al. 1998, Nature, 395, 672

Johnston, S., Feain, I. J., \& Gupta, N. 2009, The Low-Frequency Radio Universe, 407, 446

Kistler, M. D., Yuksel, H., Ando, S., Beacom, J. F., \& Suzuki, Y. 2008, arXiv:0810.1959

Komatsu, E., et al. 2009, ApJS, 180, 330

Kudritzki, R.-P., \& Puls, J. 2000, ARA\&A, 38, 613 
Kulkarni, S. R., et al. 1998, Nature, 395, 663

Leitherer, C., Robert, C., \& Drissen, L. 1992, ApJ, 401, 596

Li, W., et al. 2011, MNRAS, 412, 1441

Li, W., Chornock, R., Leaman, J., Filippenko, A. V., Poznanski, D., Wang, X., Ganeshalingam, M., \& Mannucci, F. 2011, MNRAS, 412, 1473

Li, Z.-Y., \& Chevalier, R. A. 1999, ApJ, 526, 716

Lien, A., \& Fields, B. D. 2009, Journal of Cosmology and Astro-Particle Physics, 1, 47

Lien, A., Fields, B. D., \& Beacom, J. F. 2010, Phys. Rev. D, 81, 083001

LSST Science Collaborations, et al. 2009, LSST Science Book, arXiv:0912.0201

MacFadyen, A. I., \& Woosley, S. E. 1999, ApJ, 524, 262

MacFadyen, A. I., Woosley, S. E., \& Heger, A. 2001, ApJ, 550, 410

Madau, P., della Valle, M., \& Panagia, N. 1998, MNRAS, 297, L17

Mannucci, F., Della Valle, M., \& Panagia, N. 2007, MNRAS, 377, 1229

Mokiem, M. R., et al. 2007, A\&A, 473, 603

Montes, M. J., Weiler, K. W., \& Panagia, N. 1997, ApJ, 488, 792

Munari, U., Barbon, R., Piemonte, A., Tomasella, L., \& Rejkuba, M. 1998, A\&A, 333, 159

Nakano, S., \& Aoki, M. 1997, IAU Circ., 6795, 2

Nomoto, K., Thielemann, F.-K., \& Yokoi, K. 1984, ApJ, 286, 644

Palanque-Delabrouille, N., et al. 2010, A\&A, 514, A63

Panagia, N., Van Dyk, S. D., Weiler, K. W., Sramek, R. A., Stockdale, C. J., \& Murata, K. P. 2006, ApJ, 646, 369

Papenkova, M., Li, W. D., Wray, J., Chleborad, C. W., \& Schwartz, M. 2001, IAU Circ., 7722,1

Phillips, M. M. 1993, ApJ, 413, L105

Pooley, D., et al. 2002, ApJ, 572, 932 
Price, P. A., et al. 2002, ApJ, 572, L51

Riess, A. G., Press, W. H., \& Kirshner, R. P. 1996, ApJ, 473, 88

Smartt, S. J., Eldridge, J. J., Crockett, R. M., \& Maund, J. R. 2009, MNRAS, 395, 1409

SKA Design Reference Mission, 2009, http://www. skatelescope.org/PDF/DRM_v1.0.pdf.

Sako, M., et al. 2008, AJ, 135, 348

Salpeter, E. E. 1955, ApJ, 121, 161

Soderberg, A. M., Frail, D. A., \& Wieringa, M. H. 2004, ApJ, 607, L13

Soderberg, A. M., Nakar, E., Berger, E., \& Kulkarni, S. R. 2006, ApJ, 638, 930

Soderberg, A. M., Chevalier, R. A., Kulkarni, S. R., \& Frail, D. A. 2006, ApJ, 651, 1005

Soderberg, A. M. 2007, Supernova 1987A: 20 Years After: Supernovae and Gamma-Ray Bursters, 937, 492

Stockdale, C. J., Weiler, K. W., Van Dyk, S. D., Montes, M. J., Panagia, N., Sramek, R. A., Perez-Torres, M. A., \& Marcaide, J. M. 2003, ApJ, 592, 900

Stockdale, C. J., Kelley, M., Sramek, R.A., Williams, C. L., Van Dyk, S. D., Weiler, K. W., \& Panagia, N. 2006, New Radio Supernova Results.

Stockdale, C. J., Kelley, M. T., Weiler, K. W., Panagia, N., Sramek, R. A., Marcaide, J. M., Williams, C. L. M., \& van Dyk, S. D. 2007, Supernova 1987A: 20 Years After: Supernovae and Gamma-Ray Bursters, 937, 264

The Large Synoptic Survey Telescope Collaboration 2007, Science Requirements Document, http://www.1sst.org/Science/docs/SRD.pdf

J. Tonry, (2003), Pan-STARRS Science Goals: Supernova Science, http://pan-starrs.ifa.hawaii.edu/project/science/precodr.html; http://pan-starrs.ifa.hawaii.edu.

Vink, J. S., de Koter, A., \& Lamers, H. J. G. L. M. 2001, A\&A, 369, 574

Webbink, R. F. 1984, ApJ, 277, 355

Weiler, K. W., Sramek, R. A., Panagia, N., van der Hulst, J. M., \& Salvati, M. 1986, ApJ, 301,790 
Weiler, K. W., Panagia, N., \& Sramek, R. A. 1990, ApJ, 364, 611

Weiler, K. W., Panagia, N., Montes, M. J., \& Sramek, R. A. 2002, ARA\&A, 40, 387

Weiler, K. W., van Dyk, S. D., Sramek, R. A., \& Panagia, N. 2004, New Astronomy Review, 48, 1377

Weiler, K. W., Williams, C. L., Panagia, N., Stockdale, C. J., Kelley, M. T., Sramek, R. A., Van Dyk, S. D., \& Marcaide, J. M. 2007, ApJ, 671, 1959

Weiler, K. W., Panagia, N., Sramek, R. A., van Dyk, S. D., Williams, C. L., Stockdale, C. J., \& Kelley, M. T. 2009, American Institute of Physics Conference Series, 1111, 440

Wellons, S., \& Soderberg, A. M. 2011, Bulletin of the American Astronomical Society, 43, \#337.15

Woosley, S. E. 1993, ApJ, 405, 273

Woosley, S. E., Eastman, R. G., \& Schmidt, B. P. 1999, ApJ, 516, 788

Woosley, S. E., \& Bloom, J. S. 2006, ARA\&A, 44, 507

This preprint was prepared with the AAS LATEX macros v5.2. 